\title{
LAMBDA POLARIZATION IN EXCLUSIVE ELECTRO- AND PHOTOPRODUCTION AT CLAS
}

\author{
M. D. MESTAYER* \\ Thomas Jefferson National Accelerator Facility, \\ 12000 Jefferson Ave., \\ Newport News, Va. 23606 USA \\ E-mail: mestayer@jlab.org
}

\begin{abstract}
The CLAS collaboration at JLab has recent results on $\Lambda$ polarization for both electroproduction and photoproduction of $K^{+} \Lambda$ exclusive states. I note the striking phenomenological trends in the data and discuss the underlying physics which might give rise to these phenomena; both in the context of an effective Lagrangian formalism, where the degrees of freedom are intermediate mesons and baryons, and also in the context of a simple quark picture. The quark model argument leads to the conclusion that the $s$ and $\bar{s}$ quarks are produced with spins anti-aligned, in apparent contradiction to the popular ${ }^{3} P_{0}$ model of quark pair creation in which the pair is created with vacuum quantum numbers $(J=0$ and positive parity), i.e. in an $S=1, L=1, J=0$ angular momentum state.
\end{abstract}

\section{Introduction}

One of the important goals of particle and nuclear physics is to understand strong interaction dynamics. Studying hadron production sheds light on these dynamics because it focusses on just how quark pair creation (QPC) neutralizes the strong forces which bind quarks into hadrons. Because $s \bar{s}$ creation is the lowest energy manifestation of QPC of a flavor which is not a valence quark in a nucleon, it is of special interest. By a happy circumstance, the $\Lambda$ 's decay is self-analyzing, and its assumed $\mathrm{SU}(3)$ wavefunction has all of its spin carried by the $s$-quark, so its decay distribution is directly related to the spin of its $s$-quark. Thus, we have studied $\Lambda$ polarization in exclusive production in an attempt to understand the spin-dynamics of $\mathrm{QPC}$ and its role in neutralizing the strong force field, i.e. "breaking the gluonic flux-tube".

*representing the CLAS collaboration. 
In this contribution, I will describe two recent experiments which have been published ${ }^{1,2}$ by the CLAS collaboration. The first is a measurement of induced $\Lambda$ and $\Sigma^{0}$ polarization in photoproduction of $K^{+} \Lambda$ and $K^{+} \Sigma^{0}$ from a proton target where neither beam nor target were polarized. The second is a measurement of spin transfer from a polarized electron beam to the polarized $\Lambda$, also in $K^{+} \Lambda$ production from an unpolarized proton target. Both measurements can be used to constrain hadrodynamic models in which the effective degrees of freedom are the intermediate mesons and baryons; the relevant parameters being the choice of baryons participating in $s$ and $u$-channel exchanges and the choice of mesons exchanged in the $t$-channel, the vertex coupling constants and the hadronic form factors. Alternatively, by using a qualitative, semi-classical argument, I argue that both measurements are sensitive to the spin state of the produced quark pair.

\section{Experiment Description}

These scattering experiments were performed on a hydrogen target, situated within the CLAS detector in Hall B at Jefferson Lab. These studies required the simultaneous detection of the scattered electron and the $K^{+}$, as well as the proton from the decay of the $\Lambda$. For the photoproduction experiment the beam electron was detected in the CLAS tagger, providing a measure of the photon's energy. For the electroproduction experiment, both the scattered electron as well as the $K^{+}$and proton were detected in the CLAS detector. In the latter case, the electron beam was polarized with typical values of $75 \%$.

The CLAS is a large-acceptance detector ${ }^{3}$ used to detect multi-particle final states initiated by photon or electron beams. The central element of the detector is a six-coil superconducting toroidal magnet which provides a mostly azimuthal magnetic field. Drift chambers (DC) situated before, within and outside of the magnetic field volume provide charged particle tracking. The outer detector surrounding the magnet and chambers consists of large-volume gas Cerenkov counters (CC) for electron identification, $5 \mathrm{~cm}$ thick scintillators (TOF) for triggering and charged particle identification via time-of-flight, and a lead-scintillator electromagnetic shower counter (EC) used for electron/pion separation as well as neutral particle detection and identification.

The offline event reconstruction first identified a viable electron candidate, either a tagger hit for the photoproduction experiment or a negatively 
charged track in the DC's which matched in position and energy with a hit in the outer detectors for the electroproduction case. Kaon candidates were positively charged tracks found in the DC which were spatially matched to a TOF hit. The time of flight and the measured path length yielded the particle velocity; combining this with the momentum of the DC track yielded the kaon-candidate mass. Cuts on the calculated mass and time of flight typically accepted $>99 \%$ of real kaons, but allowed some background from positively charged $\pi^{+}$'s and protons.

The data were binned in the independent kinematic variables $\cos \theta_{K}^{*}$ and $W$ where $\cos \theta_{K}^{*}$ is the cosine of the $K^{+}$angle and $W$ the total energy in the hadronic center-of-mass system. For the electroproduction experiment, we have the additional variables, $Q^{2}$, the 4-momentum squared of the virtual photon, as well as $\phi$, the angle between the leptonic and hadronic planes. Using the 4-momenta of the real (or virtual) photon and the $K^{+}$candidate, we calculated the missing mass of each event. Within each bin the missing mass spectra showed peaks at the $\Lambda$ and $\Sigma^{0}$ mass, situated on a smooth background. The $\Lambda$ and $\Sigma^{0}$ peaks were fit to templates produced with a radiative event generator and a detector simulation program, along with a low-order polynomial representing the background. For the photoproduction data, the absence of radiative corrections meant that we could use Gaussians and a polynomial background to fit the missing mass spectra.

Fiducial cuts were used to eliminate inefficient areas of the detector and the acceptance was calculated using a Monte Carlo package, GSIM, based upon the GEANT code. Our acceptance correction was combined with a bin-migration or "smearing" correction, as well as with a radiative correction factor.

\section{Photoproduction Results}

In addition to determining that the event was a $K^{+} \Lambda$ event by detecting the $K^{+}$and tagged photon, we also detected the decay proton (from the $\Lambda \rightarrow p \pi^{-}$decay mode) in order to measure the polarization of the $\Lambda$ sample.

The weak decay of the $\Lambda$ is self-analyzing, in that the angular distribution of the decay protons is proportional to $1+\alpha \cdot P \cdot \cos \theta_{p}$ where $\alpha$ is the decay analyzing power value of $0.642, P$ is the polarization of the sample of $\Lambda$ 's and $\cos \theta_{p}$ is the cosine of the angle that the proton's momentum vector makes relative to the particular axis chosen, evaluated in the $\Lambda$ rest-frame.

For photoproduction the measurement axis is chosen to be normal to the plane containing the photon and the $K^{+}, \hat{n}=\hat{q} \times \hat{p}_{K}$. Because parity 
is conserved in $K^{+} \Lambda$ production, this pseudo-vector is the only one available with which to form a Lorentz-invariant, parity-conserving term in the Lagrangian when dotted into the $\Lambda$ spinor, $\sigma$. So, for photoproduction, the polarization can only be non-zero along the normal $(\hat{n})$ axis.

The analysis proceeded in a straightforward manner: for each bin in $W$ and $\cos \theta_{K}^{*}$ an acceptance-corrected yield of protons was binned according to the cosine of the direction of the proton momentum relative to $\hat{n}$ and then fit to $1+\alpha \cdot P \cdot \cos \theta_{p}$, with the value of the polarization, $P$, being the free parameter.

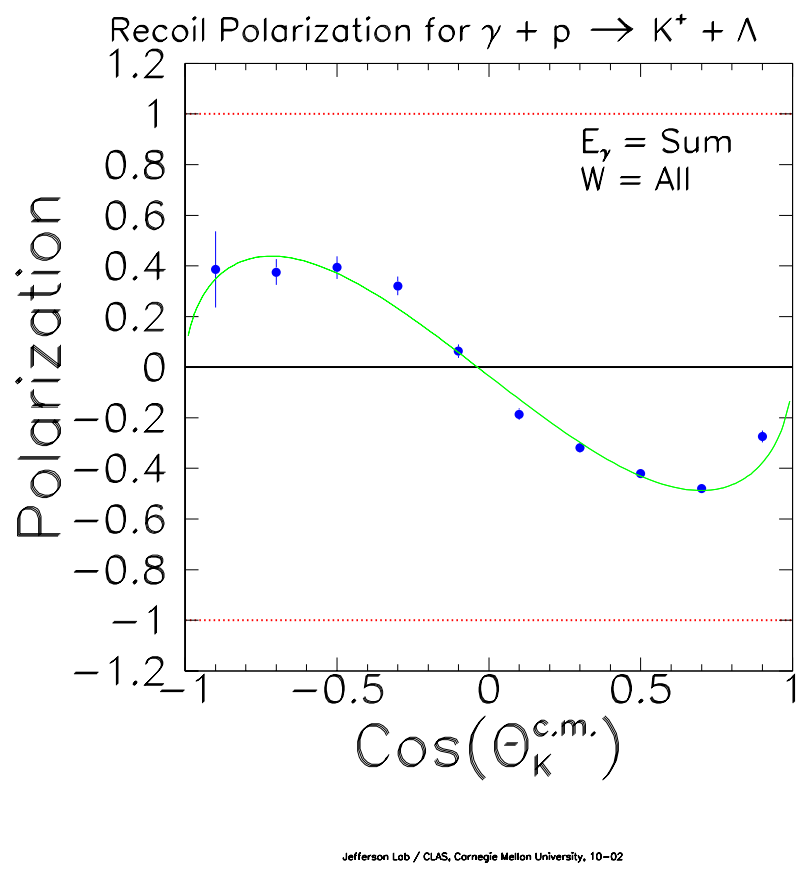

Figure 1. Recoil polarization in $\gamma p \rightarrow K^{+} \Lambda$ events averaged over all $E_{\gamma}$. The curve is a phenomelogical fit which is constrained to equal 0 at the endpoints.

To determine the polarization of the $\Sigma^{0}$ we measured the polarization of its daughter $\Lambda$ produced from the electromagnetic decay, $\Sigma^{0} \rightarrow \Lambda \gamma$. The $\Sigma^{0}$ 's polarization signal is diluted during its electromagnetic decay to the $\Lambda$ and a $\gamma$ with the daughter $\Lambda$ 's polarization being opposite in direction 
and $1 / 3$ the strength of that of the parent $\Sigma^{0}$. Thus, the coefficient has an additional factor, $\alpha_{\Sigma}=-0.333$ and the proton's angular distribution is fit to $1+\alpha_{\Sigma} \cdot \alpha \cdot P \cdot \cos \theta_{p}$. In Figs. 1 and $2, \mathrm{I}$ show the $\Lambda$ and the $\Sigma^{0}$ polarization versus $\cos \theta_{K}^{*}$ averaged over all incident photon energies.

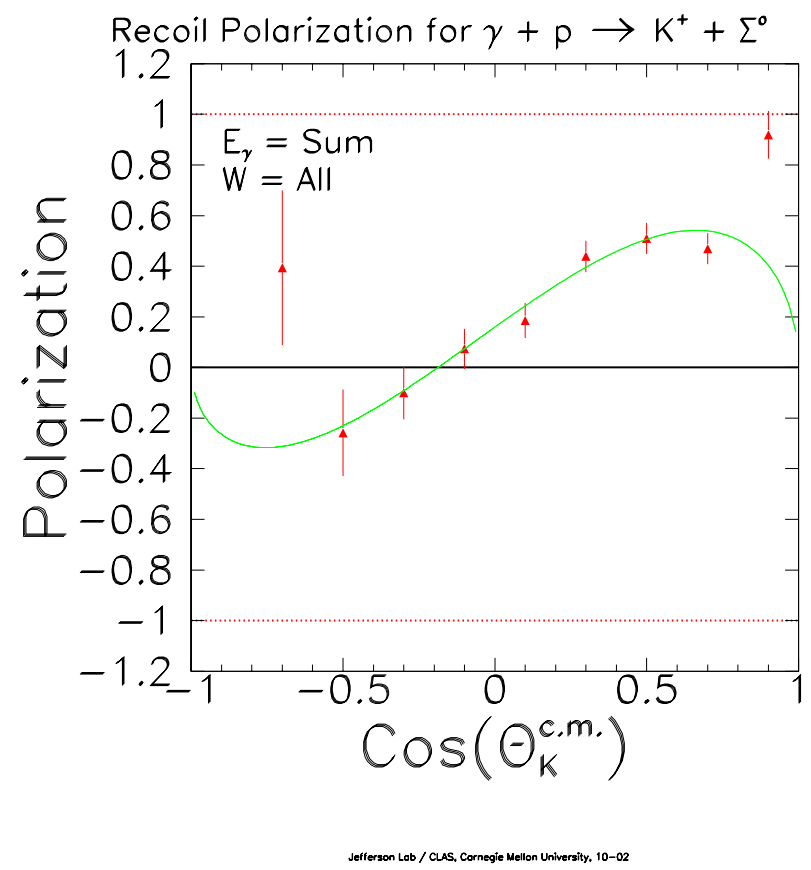

Figure 2. Recoil polarization in $\gamma p \rightarrow K^{+} \Sigma^{0}$ events averaged over all $E_{\gamma}$. The curve is a phenomelogical fit which is constrained to equal 0 at the endpoints.

First, note the simple phenomenology: the $\Lambda$ 's are negatively polarized (opposite the normal to the hadronic plane) for kaons produced in the forward hemisphere and positive for backward-going kaons. Interestingly, the $\Sigma^{0}$ polarization values are approximately equal in magnitude but opposite in sign from the $\Lambda$ values. This finding has a natural explanation within quark models if one assumes that in $K^{+}$- hyperon production there is a single underlying production mechanism which polarizes the $s$-quark. In a simple $\mathrm{SU}(3)$ model the $u d$ is in a spin- 0 , isospin- 0 state for the $\Lambda$ but in a spin-1, isospin-1 state for the $\Sigma^{0}$. This implies that the $\Lambda^{\prime}$ 's polarization is 
parallel to the $s$-quark 's spin, and that the $\Sigma^{0}$ 's polarization is anti-parallel to the $s$-quark 's spin. So, perhaps there is a common, quark model explanation for the pattern of polarization observed in $K^{+} \Lambda$ and $K^{+} \Sigma^{0}$ final states.

I speculate about the cause of this simple phenomenology as follows. Suppose that there is a spin-orbit force which polarizes the $u$-quark in a direction which is anti-parallel to $\hat{n}$. Such a phenomelogical spin-orbit force which polarizes quarks involved in strong scattering has been invoked to explain, e.g., single-spin asymmetries in $p p$ scattering where one of the protons is polarized. See the article by Zuo-tang and Boros ${ }^{4}$ for a detailed explanation. In order for the $K^{+}$to have spin-zero, the $\bar{s}$ quark's spin is selected to be parallel to $\hat{n}$. As we have just noted, we observe the $\Lambda$ 's polarization (and thus the $s$-quark 's polarization) to be anti-parallel to $\hat{n}$; thus, we infer that the $s$ and $\bar{s}$ were produced with spins anti-aligned. In the conclusion, I discuss the implications of this inference.

\section{Electroproduction Results}

For our electroproduction experiment the electron beam (and hence the virtual photon) has net circular polarization. In addition to the allowed normal polarization of the $\Lambda$ (as in real photoproduction) the existence of a new pseudo-vector in the problem (the electron's spinor) means that polarization is allowed in general along any of the three axes in the $\Lambda$ 's rest frame. However, because of limited statistics, we averaged the electroproduction data over all values of $\phi$; this integrated the normal component to zero. So, the $\Lambda$ 's polarization transfer can now only be non-zero along any two axes which are perpendicular to the hadronic plane's normal. For this presentation, I show the $x$ and $z$ components $^{5}$ of the polarization, where $z$ is parallel to the virtual photon's direction in the hadronic center-of-mass and $x$ is in the hadronic production plane, but perpendicular to $z$. Note that the polarization can in general be non-zero for both $x$ and $z$ except at the kinematic limits, $\cos \theta_{K}^{*}=+-1$ where the $x$-component is not well-defined and must be zero.

In Figure 3, I plot the $\Lambda$ polarization transfer ( $\Lambda$ polarization divided by the electron beam polarization) along $z$ (top) and $x$ (bottom) as a function of $\cos \theta_{K}^{*}$ for three values of $W$. There are several features worth noting: the polarization transfer is consistent with zero along the $x$-axis though

the models shown (which have NOT been tuned to the present data) are in general, non-zero; second, the $z$-polarization transfer is large (about 75\%) 
and is flat or shows a slow rise with $\cos \theta_{K}^{*}$; and thirdly, the polarization seems to be independent of center-of-mass energy, $W$.

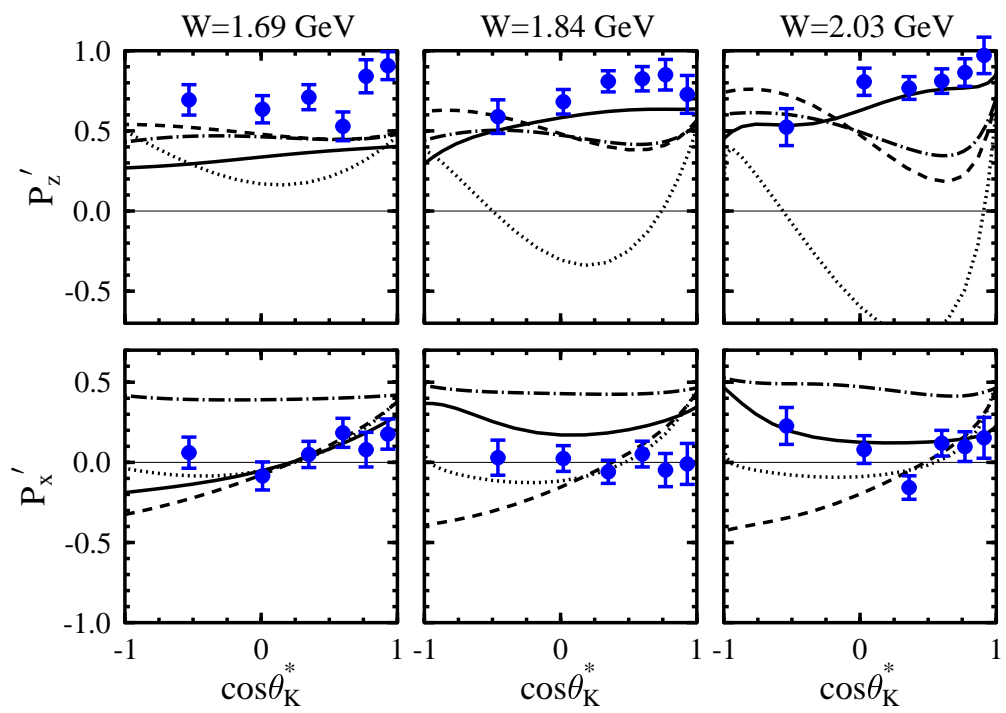

Figure 3. Transferred $\Lambda$ polarization components $P_{x}^{\prime}$ and $P_{z}^{\prime}$ plottedq versus $\cos \theta_{K}^{*}$, summed over all $Q^{2}$ and $\Phi$ for three $w$ bins centered at $1.69,1.84$ and $2.03 \mathrm{GeV}$. The curves are WJC92 (dotted), BM98 (dashed), BM02 (solid), J02 (dot-dashed), averaged over the experimental bins.

The models shown are from Williams, Ji and $\operatorname{Cotanch}^{6}$ (WJC92), from Bennhold and $\mathrm{Mart}^{7}$ (BM98 and BM02), or from Janssen et al. ${ }^{8}(\mathrm{~J} 02)$. They are all effective Lagrangian type models but with different choices for the spectra of hadrons in the $s, t$ and $u$-channels as well as different values of masses, coupling constants and form-factors. Although not shown, a recent calculation from Laget, Guidal and Vanderhaegen ${ }^{9}$ is in reasonable agreement with our data.

\section{Discussion and Conclusions}

Measured values of $\Lambda$ polarization in exclusive electro- and photoproduction are large (about $75 \%$ for the transferred polarization and reaching a maximum of about $60 \%$ for the induced polarization case). $\Lambda$ polarization has been measured in semi-inclusive $\Lambda$ production at higher energies by HERMES ${ }^{10}$, with much smaller values. This is consistent with ob- 
servations in $p p$ production: exclusive processes yield significantly larger polarizations ${ }^{11}$ than do semi-inclusive measurements.

If viewed as observables which are sensitive to interference terms between different intermediate states in a hadro-dynamic model, our measurements can serve as important constraints on the models. These constraints improve these models' reliability and hence, predictive power, in the combined experimental and theoretical search for new, previously missing, $s$-channel baryon resonances.

If viewed in terms of a semi-classical quark picture, the polarization arises after some other process polarizes a $u$-quark (either helicity conservation in the electroproduction case or a phenomelogically-motivated spin-orbit force in the case of real photoproduction). Because the kaon is a spin- 0 particle, the $\bar{s}$ is selected to be polarized opposite the polarization direction of the $u$-quark. Measuring the $\Lambda$ polarization reveals the polarization of the $s$-quark, and it is observed to be parallel to the presumed direction of the $u$-quark spin. This argument thus implies that the $s$ and $\bar{s}$ are produced with spins anti-aligned. For a similar argument which explains the $\Lambda$ polarization observed in the reaction $p p \rightarrow p K^{+} \Lambda{ }^{11}$, see the article by Boros and Zuo-tang ${ }^{4}$. We also note that this simple picture would predict that the $\Lambda$ polarization would flip sign if its mesonic partner were a spin- $1 K^{*+}$ instead of the pseudo-scalar $K^{+}$. Since semi-inclusive polarizations are presumably a sum over exclusive states, some with vector and some with scalar recoil mesons, we might expect that the net polarization is much smaller than in the case of an exclusive process.

In this semi-classical quark picture the $\Lambda$ polarization observed in $K^{+} \Lambda$ exclusive production arises from a first step which polarizes the $u$-quark followed by a QPC process in which the $s \bar{s}$ pair is produced with spins anti-aligned. This is not what is naively expected if QPC proceeds through a ${ }^{3} P_{0}$ wavefunction. The ${ }^{3} P_{0}$ state has the $q \bar{q}$ pair in an $S=0$ state, so, on average the spins are aligned $2 / 3$ of the time. Perhaps $s \bar{s}$ pairs are not produced with vacuum quantum numbers at all, but in a spectra of angular momentum states? At any rate, we hope that some theoretical work can prove or disprove our assertion that the polarization of the $\Lambda$ in exclusive final states is sensitive to the angular momentum state of the produced $s \bar{s}$ - If our assertion that the $s \bar{s}$ pair is produced with spins anti-aligned is validated, then it will provide an important constraint in modelling strong decays, and more generally, will provide insight into how the gluonic fluxtube is "broken" or neutralized during the QPC process. 


\section{Acknowledgments}

This paper represents the work of the entire CLAS collaboration, although some of the speculations are my own. I wish to recognize the important help provided by Nathan Isgur, who was alway encouraging, and who discovered the Zuo-tang and Boros article. Finally, I greatly appreciate the thorough planning and cheerful generosity of our host, Professor Hashimoto, and his colleagues.

\section{References}

1. J.W.C. McNabb et al., nucl-ex/0305028, submitted to Phys. Rev. Lett., J.W.C. McNabb thesis, Carnegie Mellon University, (2002), unpublished.

2. D.S. Carman et al., Phys. Rev. Lett. 90, 131804 (2003).

3. B. Mecking et al., it Nucl. Instr. and Methods A503, 515 (2003), and references therein.

4. Liang Zuo-tang and C. Boros Phys. Rev. D61, 117503 (2000), and references therein.

5. Another common choice of axes is $l$ and $t$, with $l$ along the $K^{+}$direction and $t$ tranverse to $l$ but in the hadronic plane.

6. R.A. Williams et al., Phys. Rev. C46, 1617 ,(1992).

7. T. Mart and C. Bennhold, Phys. Rev. C61, 012201, (2000).

8. S. Janssen et al., Phys. Rev. C65, 015201, (2002).

9. M. Guidal, J.-M. Laget and M. Vanderhaegen, hep-ph/0308131; submitted to Phys. Rev.C.

10. M. Airapetian et al., Phys. Rev. D64, 112005, 2001.

11. T. Henkes et al., Phys. Lett. B283, 155 (1992). 